\title{
Pseudomonal airway colonisation: risk factor for bronchiolitis obliterans syndrome after lung transplantation?
}

\author{
R. Vos*, B.M. Vanaudenaerde*, N. Geudens" ${ }^{\#}$, L.J. Dupont*, ${ }^{*},+$, \\ D.E. Van Raemdonck ${ }^{\#, \uparrow^{,},}$and G.M. Verleden ${ }^{*, \sigma_{,+}}$
}

ABSTRACT: Airway colonisation with Pseudomonads, especially Pseudomonas aeruginosa, is common in lung transplant (LTx) recipients. The current authors investigated whether pseudomonal colonisation affects the prevalence of bronchiolitis obliterans syndrome (BOS) after lung transplantation.

In the present retrospective study, 92 double (SS)LTx recipients (26 cystic fibrosis (CF) and 66 non-CF patients), with at least two consecutive post-operative bronchoalveolar lavage or sputum cultures evaluated for Pseudomonads, were included. Freedom of BOS was investigated in postoperatively colonised and noncolonised patients.

The current study has shown post-operative airway colonisation to be an independent risk factor for BOS stage $\geqslant 1$ and to be associated with a worse BOS stage $\geqslant 1$-free survival in univariate analysis, especially in CF SSLTx recipients. Multivariate analysis demonstrated a trend for colonisation only as an independent risk factor for BOS; however, this pointed to a possible role in the development of BOS.

In conclusion, pseudomonal airway colonisation after lung transplantation may be associated with an increased prevalence of bronchiolitis obliterans syndrome, especially in cystic fibrosis patients. Possible pathophysiological mechanisms in the development of bronchiolitis obliterans syndrome need further investigation, although the induction of neutrophilic airway inflammation seems to be its main characteristic.

KEYWORDS: Airway colonisation, bronchiolitis obliterans syndrome, lung transplantation, Pseudomonas aeruginosa

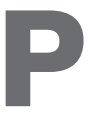

seudomonas aeruginosa is a Gram-negative, nonacid-fast, aerobic rod belonging to the genus Pseudomonas and the bacterial family of Pseudomonadaceae. Former Pseudomonas species have recently been reclassified and belong to other genera, such as Burkholderia ( $B$. cepacia, Alcaligenes xylosoxidans), Xanthomonas (Stenothrophomonas), Aeromonas, etc., which are generally referred to as Pseudomonads [1]. Airway colonisation with Pseudomonads, especially $P$. aeruginosa, is common in patients with altered pulmonary defences [2-6], but also in immunocompromised lung transplant (LTx) recipients [7-9]. Colonisation with mucoid or multiple-antibiotic resistant $P$. aeruginosa or $B$. cepacia is associated with a worse prognosis, particularly in cystic fibrosis (CF) patients [1012]. It has been extensively shown that colonisation triggers expression of diverse cytokines by structural airway cells, inducing neutrophil recruitment and thereby perpetuating a cycle of airway inflammation and destruction [13-18]. However, whether pseudomonal airway colonisation after LTx occurs secondary to the airway remodelling in bronchiolitis obliterans syndrome (BOS), the major cause of late graft failure and death in long-term survivors after LTx [19], or similarly plays a primordial role in its aetiology and progression remains elusive [7, 20-22]. The aim of the present retrospective study, therefore, was to assess the importance of pseudomonal airway colonisation for the development of BOS after LTx.

\section{MATERIALS AND METHODS}

\section{Study population}

In the present retrospective study, approved by the Ethical Review Board of the University
AFFILIATIONS

*Laboratory of Pneumology,

\#Laboratory of Experimental Thoracic

Surgery, Katholieke Universiteit

Leuven,

"Lung Transplantation Unit,

Depts of ${ }^{+}$Respiratory Disease, and

${ }^{\S}$ Thoracic Surgery, University

Hospital Gasthuisberg, Leuven,

Belgium.

CORRESPONDENCE

G.M. Verleden

University Hospital Gasthuisberg

Lung Transplantation Unit

49 Herestraat

B3000 Leuven

Belgium

Fax: 3216346803

E-mail: geert.verleden@

uz.kuleuven.ac.be

Received:

October 012007

Accepted after revision:

January 182008

STATEMENT OF INTEREST

Statements of interest for R. Vos,

B.M. Vanaudenaerde, L.J. Dupont and

the study itself can be found at

www.erj.ersjournals.com/misc/

statements.shtml 
Hospital Gasthuisberg (Leuven, Belgium), freedom of BOS was investigated in colonised and noncolonised double (SS)LTx recipients, transplanted between January 2000 and December 2005, who underwent routine longitudinal follow-up at the outpatient clinic, as previously described [17].

Inclusion criteria were as follows: at least two consecutive positive bronchoalveolar lavage (BAL) or sputum cultures for Pseudomonads, taken after the first three post-operative weeks and with a minimum of 4 weeks and a maximum of 6 months in between, during which period, as at the time-point of sampling, the patient was clinically stable, immunosuppressive treatment remained unchanged and no new antibiotics (including azithromycin) were given.

Exclusion criteria were as follows: retransplantation; singlelung, heart-lung or multi-organ transplantation; airway stenting; post-operative survival $<6$ months; follow-up $<1$ yr or loss of follow-up; absence of suitable culture data; concomitant infection or acute rejection (AR) either clinically or on transbronchial biopsies (TBB; exclusion of possible subclinical $\mathrm{AR}$ ) and suture problems (making the diagnosis of BOS difficult); and, finally, post-transplant lymphoproliferative disorder, malignancies or diffuse alveolar damage.

\section{BAL and sputum cultures}

For routine bacteriological cultures, $100 \mu \mathrm{L}$ of the obtained sample was serially diluted on five different media (blood, mannitol salt, MacConkey, Haemophilus-selective and Sabouraud agar). Additional media (e.g. for anaerobes, Legionella and mycobacteria) were used depending on clinical suspicion. In the current authors' centre, a semiquantitative distinction was made between heavy $(+++)$, mild $(+)$ or no growth of the obtained cultures. The presence of at least one bacterial colony after $48 \mathrm{~h}$ of incubation was considered to be significant.

To prevent sampling biases, all post-transplant BAL and sputum cultures of each recruited patient were evaluated. The post-operative day of the first of both positive cultures was taken as the 'time-point of colonisation'. Patients with a single positive pseudomonal culture were not included and were regarded as having no suitable culture data. Similarly, when evaluating all cultures in the 6 months preceding LTx, preoperative colonisation was defined as two consecutive positive BAL or sputum cultures as outlined above, or as pseudomonal growth from cultured biopsies of either of the native lungs at the time of LTx.

Infection was defined as a positive (myco-) bacterial or fungal culture or positive viral culture, immunoassay or molecular test on blood or BAL, combined with clinical findings (fever, and the need for antibiotic, antifungal or antiviral treatment), measurement of $\mathrm{C}$-reactive protein $\left(\mathrm{CRP} ;>5 \mathrm{mg} \cdot \mathrm{dL}^{-1}\right)$, radiological examination (new radiological infiltrates) and, if available, histological examination of TBB.

\section{Bronchoscopy with BAL and TBB}

Bronchoscopic procedures with BAL for microbiological and virological assessment, as well as quantification of BAL cell differentials and interleukin (IL)-8 protein levels, were performed as previously described [17]. TBB were taken routinely at days 21 and 90, or if AR or infection was suspected based on clinical, radiological or pulmonary function criteria. TBB specimens were examined by a pathologist skilled in LTx according to the International Society for Heart and Lung Transplantation (ISHLT) guidelines [23].

\section{Lung function measurement}

Forced expiratory volume in one second (FEV1) was measured according to American Thoracic Society (ATS) criteria [24]. BOS was diagnosed based on FEV1 monitoring, according to the ISHLT working formulation [25]. Only BOS stages $\geqslant 1$ were considered to assess BOS-free survival in the present study [26]

\section{Therapeutic regimen}

Conventional pre- and post-operative prophylactic and immunosuppressive treatments are outlined in the supplementary material. Standard immunosuppressive regimen was not altered when colonisation was present. Pre-operative treatment with inhaled colistin for pseudomonal airway colonisation in CF patients was post-operatively continued for 2 3 months to prevent recolonisation. Antibiotic treatment for bacterial infection after LTx was guided using bacteriological cultures; airway colonisation, however, was not treated by antibiotics, except in those patients with recurrent infections due to multiresistant Pseudomonads in whom maintenance therapy with inhaled colistin was started. Azithromycin treatment for chronic rejection was not started until potentialBOS stage $\geqslant 0$-p was diagnosed.

\section{Statistical analysis and data management}

Survival analysis was performed with the Chi-squared test. Contingency tables were evaluated using the Fisher's Exact test, nonparametric correlation using the Spearman rank test, and the Mann-Whitney U-test was used where appropriate. For multivariate analysis, clinically relevant variables that significantly correlated with BOS in univariate analysis (Spearman rank test) were subsequently entered in a stepwise, multivariate, logistic regression model. Results of the data are presented as median (interquartile range) or as percentages.

\section{RESULTS}

\section{Study population}

Out of the total 126 SSLTx recipients receiving transplants between January 2000 and December 2005 at the present authors' centre, 92 SSLTx recipients (26 CF and 66 non-CF patients) were included in the current study based on the aforementioned inclusion and exclusion criteria. Patients' characteristics are given in table 1. Exclusion criteria for the excluded patients $(n=34)$ are listed in table 2 . In the included SSLTx recipients, independent clinical variables that significantly correlated with the development of BOS were both the total number and grade of histologically confirmed AR and lymphocytic bronchitis/bronchiolitis, the total number of pulmonary (noncytomegalovirus (CMV)) infections requiring hospital admission and intravenous antibiotic treatment, pseudomonal airway colonisation and treatment with tacrolimus (table 3) [27]. Treatment with tacrolimus was somewhat associated with both the total number of AR $(p=0.06)$ and CMV infections $(p=0.09$; Spearman rank test), reflecting the fact that immunosuppressive treatment with cyclosporine was most often switched to tacrolimus in these conditions. 

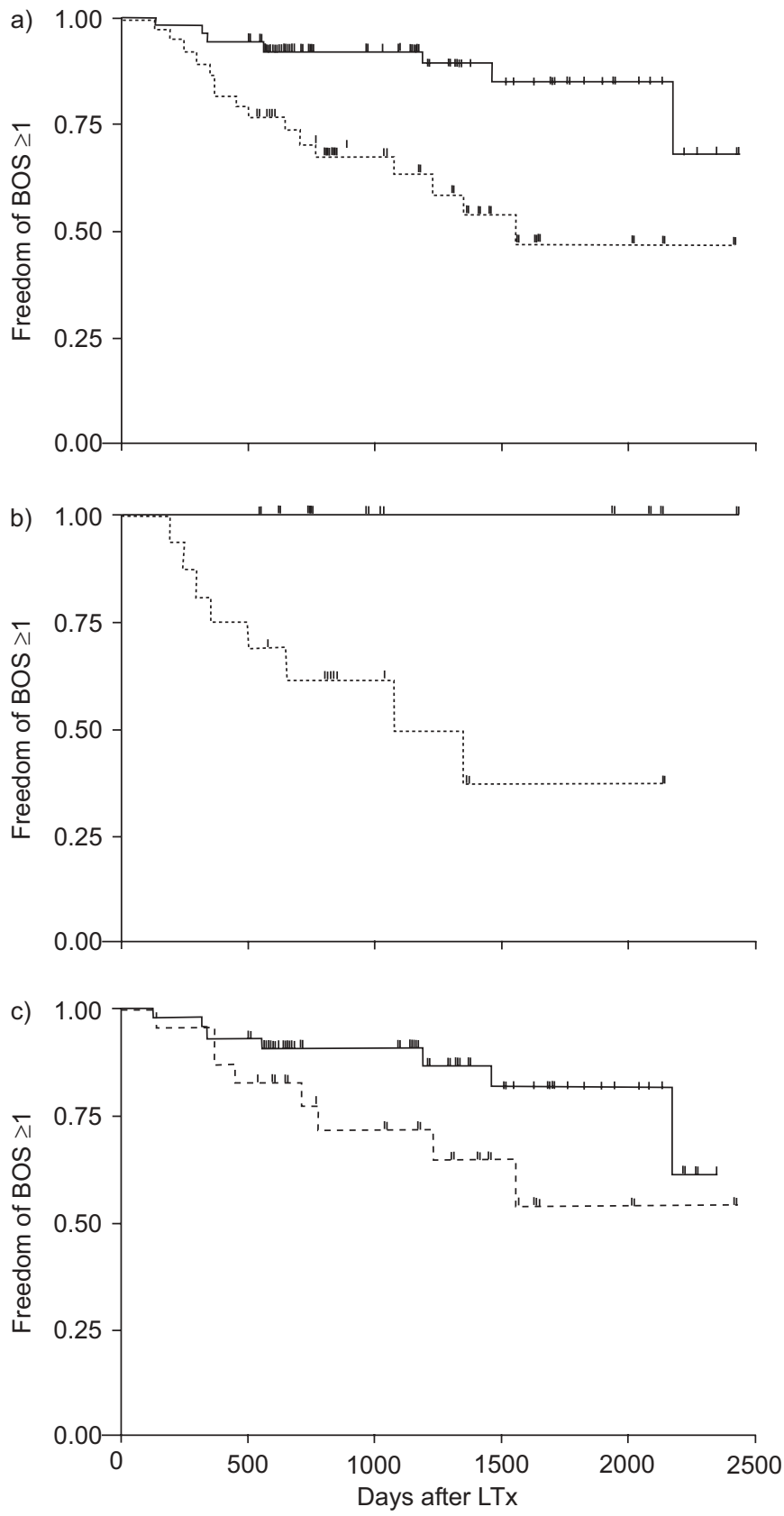

FIGURE 1. Freedom of bronchiolitis obliterans syndrome (BOS). a) Total group ( $n=92)$ : BOS $\geqslant 1$-free survival of colonised $(n=39)$ compared with noncolonised $(n=53)$ double lung transplant (SSLTX) recipients. $p=0.001$. b) Cystic fibrosis (CF) subgroup ( $n=26$ ): $B O S \geqslant 1$-free survival of colonised $(n=16)$ compared with noncolonised $(n=10)$ SSLTx recipients. $p=0.013$. c) Non-CF group ( $n=66)$ : BOS $\geqslant 1$-free survival of colonised $(n=23)$ compared with noncolonised ( $n=43)$ SSLTX recipients. - - noncolonised subjects; --.--.. colonised subjects. a) $p=0.001$; b) $p=0.013$; c) $p=0.079$.

\section{Airway colonisation and inflammation}

A total of 756 BAL samples (median 8 (range 5-10) per patient) and 1,184 sputum samples (9 (5-15) per patient) were analysed to determine post-transplant airway colonisation in the 92 included SSLTx recipients. BAL sample numbers per patient were generally comparable between colonised and noncolonised patients (9 (5-11) versus 7 (5-10); non significant (NS)),
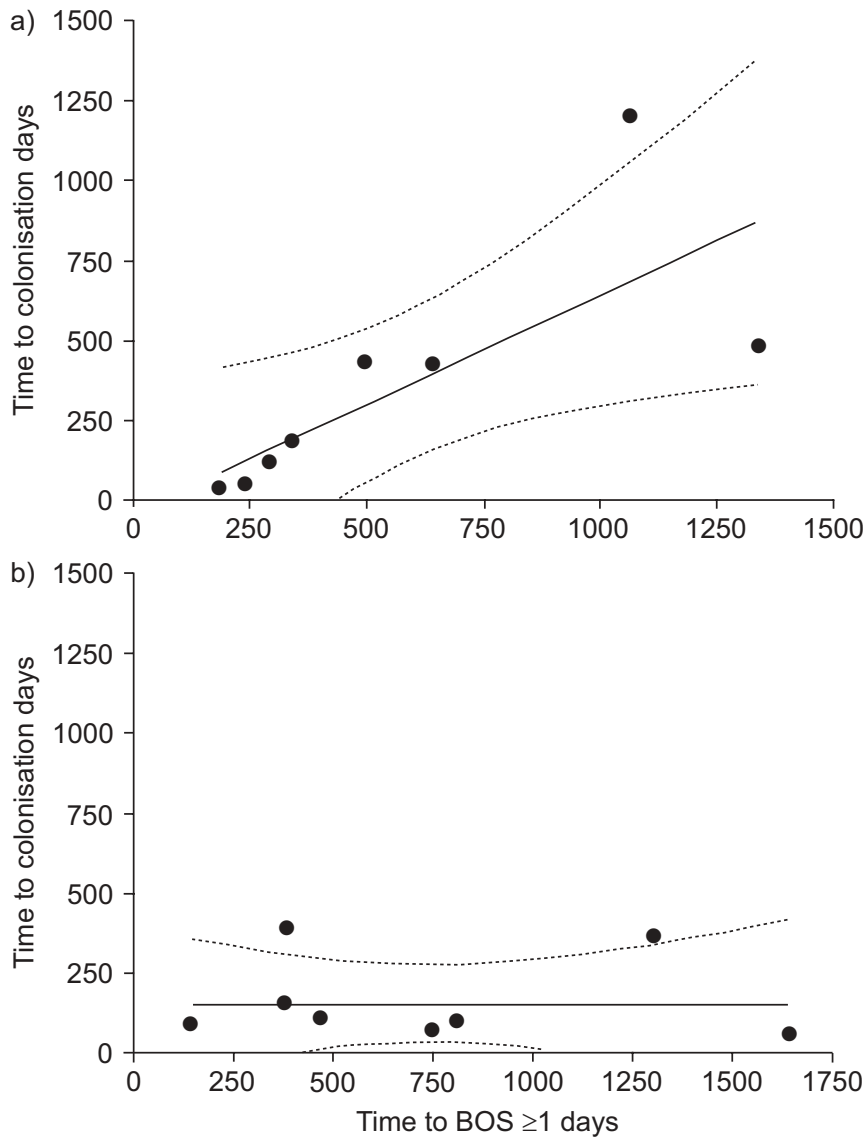

FIGURE 2. Correlation between onset of colonisation and bronchiolitis obliterans syndrome $(B O S) \geqslant 1$ Spearman rank correlation (with regression line $(-)$ and $95 \%$ confidence intervals (-----) calculated by univariate regression model) between the post-operative day of colonisation (days) and onset of BOS $\geqslant 1$ (days) in a) cystic fibrosis (CF) double lung transplant (SSLTx) recipients $(n=8$ Spearman $r=0.95, p=0.001$, power for $\alpha 0.05$ (two-sided)=0.989) and $b$ ) non-CF SSLTx recipients ( $n=8, p=$ not significant). $\bullet$ : represents one subject.

sputum sample numbers per patient were higher in colonised patients $(13(7-24)$ versus $8(4-11) ; p=0.004)$. Of all BAL samples, $21 \%$ were regarded as being colonised, whereas $25 \%$ of all sputum samples were positive for Pseudomonads.

Almost all CF SSLTx recipients were pre-operatively colonised with Pseudomonads, whereas fewer regained post-operative colonisation. Conversely, a minority of the non-CF SSLTx recipients was pre-operatively colonised, but post-operatively this number almost tripled (table 1). Time to pseudomonal (re)colonisation after LTx was comparable between CF and non-CF SSLTx recipients $(\mathrm{p}=\mathrm{NS}$; table 1$)$; pre-operative as well as post-operative colonisation was mainly due to $P$. aeruginosa (table 1). Post-operatively, coincident colonisation with nonpseudomonal species was seen in both the CF (Escherichia coli, $\mathrm{n}=2$ ) and the non-CF SSLTx recipients (Serratia sp., $\mathrm{n}=3$; Enterobacter $s p ., \mathrm{n}=1$ ), and, in both groups, intermittent concurrent colonisation of $P$. aeruginosa with other Pseudomonads was more frequent and most often seen with Stenotrophomonas spp. Pulmonary (non-CMV) infections (including those caused by $P$. aeruginosa) after the onset of colonisation were more frequent in 
TABLE 1 Characteristics of patients included in the present study ${ }^{\#}$

\begin{tabular}{|c|c|c|c|}
\hline & Total group & $\mathrm{CF}$ & Non-CF \\
\hline Subjects & 92 & 26 & 66 \\
\hline Sex $M / F$ & $52 / 40$ & $14 / 12$ & $38 / 28$ \\
\hline \multicolumn{4}{|l|}{ Pre-LTx diagnosis } \\
\hline$\alpha_{1}$-Antitrypsin deficiency & 7 & & 7 \\
\hline $\mathrm{CF}$ & 26 & 26 & \\
\hline Emphysema (COPD) & 31 & & 31 \\
\hline Pulmonary arterial hypertension & 6 & & 6 \\
\hline Pulmonary fibrosis & 13 & & 13 \\
\hline BOS & 2 & & 2 \\
\hline Other & 3 & & 3 \\
\hline Stenotrophomonas & 4 & 1 & 3 \\
\hline Post-LTx colonisation with Pseudomonads & 39/92 (42.4) & $16 / 26(61.5)$ & 23/66 (34.8) \\
\hline Alcaligenes & 1 & 1 & \\
\hline Burkholderia & 1 & 1 & \\
\hline Pseudomonas & 35 & 14 & 21 \\
\hline Stenotrophomonas & 2 & & 2 \\
\hline Time of follow-up days & $1213(718-1736)$ & 1126 (779.5-2033) & $1255(671.5-1686)$ \\
\hline Time to colonisation with Pseudomonads days & $131(79-421)$ & $121(46-445)$ & $179(88-396)$ \\
\hline Interval colonisation to BOS stage $\geqslant 1$ days & $196.5(79-672.5)$ & $181.5(122-218.5)$ & $509(37-784.5)$ \\
\hline \multicolumn{4}{|l|}{ Time to $B O S$ stage $\geqslant 1$ days } \\
\hline Time to inhaled colistin treatment days & $9.5(2-15.5)$ & $9(2-12.5)$ & $200(-)$ \\
\hline Duration of colistin treatment days & $80(64.5-134.5)$ & $76(58.5-93)$ & $239(-)$ \\
\hline \multicolumn{4}{|l|}{ Prevalence of colistin treatment } \\
\hline All patients & 20/92 (21.7) & $17 / 26(65.4)$ & $3 / 66(4.5)$ \\
\hline Noncolonised patients & 6/53 (11.3) & $6 / 10(60)$ & $0 / 43(0)$ \\
\hline Colonised patients & 14/39 (35.9) & $11 / 16(68.8)$ & 3/23 (13) \\
\hline Time to azithromycin treatment days & $399(131-761)$ & $493(378-597.5)$ & $240.5(105-908)$ \\
\hline \multicolumn{4}{|l|}{ Prevalence of azithromycin treatment } \\
\hline All patients & 35/92 (38) & $9 / 26(34.6)$ & 26/66 (39.4) \\
\hline Noncolonised patients & 13/53 (24.5) & $0 / 10(0)$ & 13/43 (30.2) \\
\hline Colonised patients & 22/39 (56.4) & 9/16 (56.3) & 13/23 (56.5) \\
\hline \multicolumn{4}{|l|}{ Mortality } \\
\hline All patients & 9/92 (9.8) & $2 / 26(7.7)$ & $7 / 66(10.6)$ \\
\hline Noncolonised patients & $3 / 53(5.7)$ & 0/10 (0) & $3 / 43(6.9)$ \\
\hline Colonised patients & 6/39 (15.4) & 2/16 (12.5) & $4 / 23(17.4)$ \\
\hline
\end{tabular}

Data are presented as n, median (range) or n/n (\%). CF: cystic fibrosis; (SS)LTx: (double) lung transplant; M: male; F: female; COPD: chronic obstructive pulmonary disease; BOS: bronchiolitis obliterans syndrome. ${ }^{*}$ : patient characteristics are given for all included SSLTx recipients ( $\left.n=92\right)$, composed of CF and non-CF SSLTx recipients. 


\begin{tabular}{|c|c|c|c|c|}
\hline \multirow{2}{*}{$\begin{array}{l}\text { TABLE } 2 \\
\text { Group }\end{array}$} & \multicolumn{4}{|c|}{$\begin{array}{l}\text { Characteristics of patients excluded from the } \\
\text { present study }\end{array}$} \\
\hline & & Total group & CF & Non-CF \\
\hline Subjects & & 34 & 6 & 28 \\
\hline \multicolumn{5}{|c|}{ Exclusion criterion } \\
\hline \multicolumn{2}{|c|}{ Suture problems } & 8 & & 8 \\
\hline \multicolumn{2}{|c|}{ Early post-operative death } & 7 & & 7 \\
\hline \multicolumn{2}{|c|}{ Absence of suitable culture data } & 6 & 3 & 3 \\
\hline \multicolumn{2}{|c|}{ Post-operative malignancies } & 3 & & 3 \\
\hline \multicolumn{2}{|c|}{ Neuromuscular disease } & 3 & & 3 \\
\hline \multicolumn{2}{|c|}{ Follow-up in another country } & 2 & 1 & 1 \\
\hline \multicolumn{2}{|c|}{ Multi-organ transplantation } & 1 & 1 & \\
\hline \multicolumn{2}{|c|}{ Retransplantation } & 1 & & 1 \\
\hline \multicolumn{2}{|c|}{ Airway stenting } & 1 & 1 & \\
\hline \multicolumn{2}{|c|}{ Chylothorax } & 1 & & 1 \\
\hline \multicolumn{2}{|c|}{ Diffuse alveolar damage } & 1 & & 1 \\
\hline
\end{tabular}

colonised compared with noncolonised SSLTx recipients, and this was true for both CF and non-CF SSLTx recipients $(\mathrm{p}=0.07$ and $\mathrm{p}=0.04$, respectively).

Inhaled colistin as an early post-operative prophylactic treatment was used only in CF SSLTx recipients, whereas maintenance therapy with colistin via nebulisation was used later on in three non-CF SSLTx recipients because of recurrent infections due to multi-resistant Pseudomonads (table 1). Time to recolonisation was comparable between patients with or without prophylactic colistin: 111 (43-422) versus 94 (46$321)$ days $(\mathrm{p}=\mathrm{NS})$. Azithromycin treatment for BOS stage $\geqslant 0$ $p$ was initiated at comparable post-operative days in CF and non-CF SSLTx recipients $(\mathrm{p}=\mathrm{NS})$, as well as in colonised and noncolonised patients ( $\mathrm{p}=\mathrm{NS}$; table 1$)$. The prevalence of azithromycin treatment was comparable in $\mathrm{CF}$ and non-CF SSLTx recipients; however, it was generally higher in colonised compared with noncolonised patients (table 1). After the onset of colonisation, both BAL neutrophilia and IL-8 protein levels were generally higher in colonised patients, and both decreased to comparable levels between colonised and noncolonised patients after azithromycin treatment was begun (see figure in supplementary material).

\section{Freedom of BOS}

In general, a worse BOS $\geqslant 1$-free survival could be demonstrated in colonised patients $(\mathrm{p}=0.001$; fig. $1 \mathrm{a}$, table 1$)$, resulting in an odds ratio of 4.57 (95\% confidence interval (CI) 1.65-12.67; $\mathrm{p}=0.003)$ in univariate analysis and 3.077 (95\% CI 0.95-9.99; $\mathrm{p}=0.061$ ) in multivariate analysis (table 3 ). Time to onset of BOS $\geqslant 1$ was comparable in colonised versus noncolonised patients $(\mathrm{p}=\mathrm{NS}$; table 1). Pseudomonal colonisation after the onset of BOS $\geqslant 1$ occurred in none of the patients and preceded the onset of BOS stage $\geqslant 1$ by 181.5 (122-218.5) days in CF and 509 (37$784.5)$ days in non-CF SSLTx recipients $(\mathrm{p}=\mathrm{NS}$; table 1$)$. Of the CF SSLTx recipients who developed BOS stage $\geqslant 1$, all were colonised (table 1), resulting in a worse BOS $\geqslant 1$-free survival of colonised patients $(\mathrm{p}=0.013$; fig. $1 \mathrm{~b})$ and an odds ratio of 21.00

\begin{tabular}{llc} 
TABLE 3 & $\begin{array}{l}\text { Clinical correlates for bronchiolitis obliterans } \\
\text { syndrome (BOS) in single and multivariate } \\
\text { analyses models\# }\end{array}$ \\
& $\begin{array}{c}\text { r or odds ratio } \\
\mathbf{( 9 5 \% ~ C l )}\end{array}$ & p-value \\
& & \\
\hline Single analysis & 0.48 & $<0.0001^{*}$ \\
Acute rejection & 0.47 & $<0.0001^{*}$ \\
Lymphocytic bronchitis/bronchiolitis & 0.47 & $<0.0001^{*}$ \\
Pulmonary (non-CMV) infections & 0.32 & $0.002^{*}$ \\
Colonisation & 0.21 & $0.044^{\star}$ \\
Immunosuppressive treatment (tacrolimus) & 0.06 & 0.573 \\
Time of ischaemia & 0.02 & 0.874 \\
CMV pneumonitis & -0.01 & 0.904 \\
Sex & -0.15 & 0.165 \\
Age & & \\
Multivariate analysis & $3.08(0.95-9.99)$ & 0.061 \\
Colonisation & & \\
\hline
\end{tabular}

Cl: confidence interval; CMV: cytomegalovirus. Clinical correlates for BOS in univariate analysis (Spearman rank test) were subsequently included in a stepwise multivariate analysis (multivariate logistic regression), correcting each correlate for the former, to determine if colonisation is an independent risk factor for the development of BOS. ${ }^{*}: n=92 ;{ }^{~}: p<0.005 .{ }^{*}: p<0.05$

(95\% CI 1.05-418.80; $\mathrm{p}=0.009$ (univariate analysis)). A significant correlation between the onset (post-operative day) of colonisation and BOS stage $\geqslant 1$ could be demonstrated in CF SSLTx recipients who developed BOS $(n=8 ; r=0.95, p=0.001$; fig. 2). In non-CF SSLTx recipients, no difference in $B O S \geqslant 1$-free survival was observed in colonised patients, although a trend was seen $(p=0.079$ (univariate analysis); table 1 , fig. $1 c)$.

\section{Overall survival}

Actuarial overall survival only demonstrated a trend for a worse survival in colonised patients $(p=0.068$; fig. 3 ). Of the two deceased CF SSLTx recipients, both had developed BOS (stages 2 and 3, respectively) and both were colonised; however, both patients died of non-Pseudomonal sepsis. Of the non-CF SSLTx recipients, two colonised patients (both in BOS stage 1) died because of pulmonary P. aeruginosa infection complicated by alveolar bleeding or bronchovascular fistulisation; another colonised patient (in BOS stage 3) died because of end-stage chronic allograft rejection and one (in BOS stage 2) of respiratory Scaedosporium infection. Two noncolonised patients (both in BOS stage 0) died of acute heart failure and one (in BOS stage 2) of post-transplant lymphoproliferative disorder.

\section{DISCUSSION}

In the current study, the importance of pseudomonal airway colonisation for the development of BOS after LTx was evaluated. Post-operative airway colonisation was demonstrated to be an independent risk factor for BOS $\geqslant 1$ and was associated with an increased prevalence of BOS $\geqslant 1$ in univariate analysis, resulting in a worse BOS-free survival, especially in CF SSLTx recipients. Multivariate analysis demonstrated only a trend for colonisation as an independent 


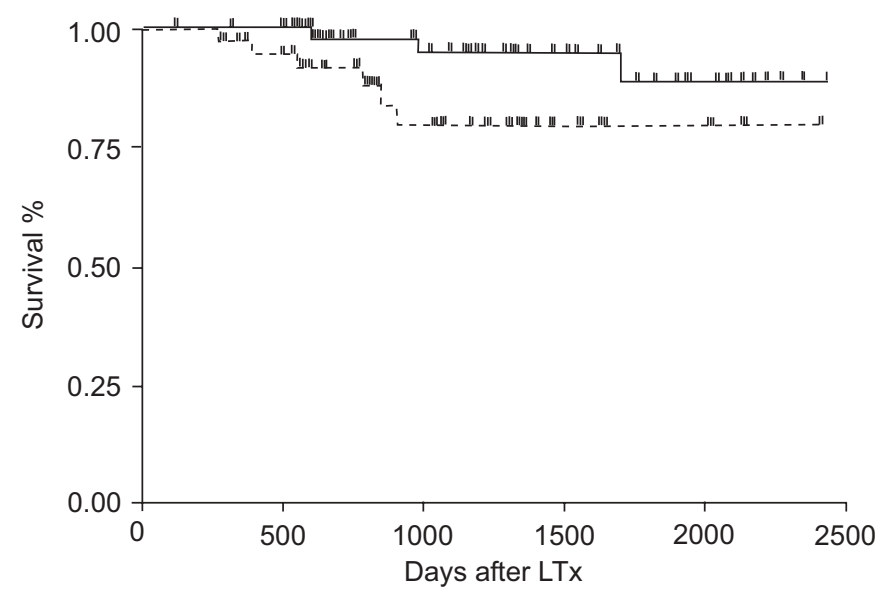

FIGURE 3. Overall survival of total number of double lung transplant (SSLTx) recipients ( $n=92)$, comparing overall survival of colonised (------; $n=39$ ) with noncolonised (—; $n=53$ ) SSLTx recipients. The survival curve was censored to survival at 6 months. $p=0.068$.

risk factor for $\operatorname{BOS}(\mathrm{p}=0.06)$, pointing to a possible role in the development of BOS.

The importance of airway colonisation in LTx remains difficult to investigate, since there is currently no consensus on an appropriate definition in this setting. Even in the CF population, with a high prevalence of colonisation, there is no consensus on the appropriate definition $[5,6]$. To differentiate colonisation from infection, protected brush catheter cultures (regarded as the 'gold standard'), quantitative BAL and sputum cultures are being used, although not consistently in identical settings, making comparisons between studies difficult [2-5, 28-33]. Regular BAL, which has a high diagnostic accuracy for the detection of lower airway inflammation, is also a generally accepted tool to assess airway inflammation [28-33]. Hence, in the present study, colonisation was defined as isolation of Pseudomonads from at least two consecutive BAL or sputum cultures, as determined by the aforementioned criteria.

$P$. aeruginosa has evolved into one of the most common and refractory nosocomial pathogens of immunocompromised patients due to its resistance to many antibiotics and a pre-dilection to colonise surfaces, especially injured epithelium, in a biofilm form, orchestrated by intercellular 'quorumsensing' signalling molecules (QSM) [34, 35]. The endobronchial presence of QSM was demonstrated in both colonised CF patients [36-39] and in clinically stable, though possibly colonised, LTx recipients [40]. QSM have an intrinsic immunomodulatory capacity resulting in suppressed T-cell proliferation, neutrophil chemotaxis and chemokine (especially IL-8) or cytokine release from bronchial epithelial cells [41-44]. As the present author's have previously shown that transient airway colonisation after LTx is associated with airway inflammation [17], it is conceivable that chronic airway colonisation with $P$. aeruginosa causes IL-8-dependent neutrophilic airway inflammation (as confirmed by the present authors' current data; see supplementary material), eventually leading to a self-perpetuating cycle of airway damage and, subsequently, airway remodelling, as seen in chronic obstructive pulmonary disease [2],
CF [13-15] and bronchiectasis [45]. Since neutrophilic airway inflammation is accepted as the main characteristic of chronic allograft rejection in LTx [46-48], it can be presumed that persistent airway colonisation with Pseudomonads facilitates the development of BOS after LTx.

In the LTx setting, respiratory epithelial injury could be due to allograft preservation injury, reperfusion injury or acute cellular rejection, possibly explaining the high prevalence of $P$. aeruginosa colonisation. Pre-operatively colonised patients (mainly CF patients) seem to be particularly at risk, possibly because the resident bacteria in the upper respiratory tract and sinuses have a direct and early access to the healing allograft. The significant correlation between the post-operative day of (re)colonisation and the time to onset of BOS in CF SSLTx recipients in the current study may support the hypothesis that early colonisation, in particular, is detrimental for the evolution to BOS. As a consequence of the latter and another study wherein CF patients underwent hygienic sinus surgery and nasal care post-LTx, resulting in a lower incidence of colonisation and a trend for a lower incidence of BOS after LTx [49], an early prophylactic or even preventative therapy for colonisation in LTx recipients could be warranted. However, the current study was not designed to investigate this question, and early post-operative prophylactic treatment with inhaled colistin did not seem to prevent recolonisation in CF SSLTx recipients. Macrolide therapy with azithromycin, which inhibits not only the production of cytokines and oxidative stress in structural airway cells [50], but also of bacterial virulence factors, such as proteases and QSM [51-53], could, therefore, be a theoretical option in these patients. Although there was a remarkably higher prevalence of azithromycin treatment in colonised patients in the current study, in most cases, this was not started until BOS 0-p had been diagnosed, and, therefore, colonisation had often already had occurred.

Some remarks can be made concerning the present study. First, the main limitation of the study is its retrospective design, the relatively small number of patients and time of sampling. The first days or weeks after transplantation may be a critical time for (re)colonisation, especially in CF patients, yet it is difficult to classify cultures obtained in this time frame to be either 'colonised' or not, given that during the early post-operative phase, serum CRP levels are generally elevated and all transplant patients are receiving antibiotic prophylaxis. Only cultures obtained after discharge of the transplanted patient were therefore used for evaluation, possibly explaining why actual time to colonisation in the CF and non-CF recipients is comparable. When the culture data during this post-operative period were reanalysed, BAL/sputum cultures positive for Pseudomonads were present in most (12 out of 16) colonised CF patients after a median of 2 days (range 1-7) post-LTx, whereas in only two out of 23 colonised non-CF patients were Pseudomonads cultured in this period before discharge (median 8.5 days), suggesting earlier colonisation in $\mathrm{CF}$ compared with non-CF patients. Secondly, the working definition of colonisation reflects pseudomonal colonisation of not only the lower airway tract, but of the entire tracheobronchial tree; however, an association between lower airway colonisation and upper airway bacterial load and IL-8 levels has been recently demonstrated [54]. As airway 
colonisation cannot always be detected using solely BAL or sputum as surveillance tools, both were combined to increase the sensitivity of detection, although quantitative cultures or measurement of pseudomonal antibody levels were not performed. The stronger association with $\mathrm{BOS}$ in the $\mathrm{CF}$ subgroup could be partly due to this methodological approach. The semi-quantitatively reporting of bacterial growth on the obtained cultures in the current authors' centre, nevertheless, is of no importance regarding the routine follow-up, immunosuppressive and prophylactic treatment after LTx, because there is no difference in follow-up or treatment between recipients with heavy, mild or no growth in the absence of clinically overt inflammation, as outlined previously. Since clinical data were used to discriminate infection from true colonisation after discharge, and comparable sample numbers of (pre- and post-transplant) BAL and sputum cultures were evaluated for each patient in the different groups, sampling biases were avoided and the probability of misclassification (i.e. considering colonisation to be present when it is not, or vice versa) reduced. Furthermore, including only SSLTx recipients prevented infection or 'spill-over' contamination of the native lung as a confounder. Although the current authors did not adjust their analysis specifically for $P$. aeruginosa infections (or its antibiotic treatment) after inclusion, pulmonary (non-CMV) infections in general (including those caused by $P$. aeruginosa) were more frequent in colonised patients, which might ultimately result in worse BOS $\geqslant 1$-free survival. Nonetheless, it is conceivable that colonisation not only induces persistent low-grade airway inflammation, but may also be associated with an increased risk of infections thereafter. Even though BOS stage $\geqslant 1$ is a rather late sign of airway involvement, only BOS stages $\geqslant 1$ were considered to assess BOS-free survival in the present study, as they are a more robust end-point for analysis than BOS stage $0-p$, which has a relatively low positive predictive value for reaching $\mathrm{BOS} \geqslant 1$ within 1 yr of developing BOS 0p [26]. Even so, it is possible that colonisation predominantly occurred in patients who had already evolved to BOS $0-p$, and thus already had bronchiolar lesions. Colonisation after the onset of BOS $0-p$, however, occurred in only two CF patients, and, in either case, within days of BOS $0-p$ being diagnosed, which may suggest that colonisation was already present before the onset of BOS $0-p$, but had been underestimated. Finally, the fact that overall survival showed only a trend for worse survival in colonised recipients, despite the higher prevalence of BOS, might be due to the fact that only SSLTx recipients were included in the present study, and they generally had a better overall survival after LTx, specifically after the onset of BOS, than single lung recipients [55]. This may explain the failure to show a significant difference in survival outcomes after the onset of BOS within the time-frame of follow-up in the current study (median $3.3 \mathrm{yrs}$ ). Furthermore, after the onset of BOS, therapeutic interventions (as for instance the start of azithromycin therapy) might stabilise further FEV1 decline, postponing mortality due to respiratory failure because of BOS.

In conclusion, pseudomonal airway colonisation after lung transplant may be a risk factor for the development of bronchioloitis obliterans syndrome, especially in cystic fibrosis patients. Its possible role and pathophysiological mechanisms in the development of bronchioloitis obliterans syndrome need further investigation, yet the induction of neutrophilic airway inflammation seems to be the main characteristic. However, to confirm the present findings, more (preferably multicentre) studies on a larger patient population are required, necessitating a more intense vigilance for and regular, standardised assessment of airway colonisation in the follow-up of lung transplant recipients.

\section{ACKNOWLEDGEMENTS}

The authors wish to give their sincere thanks to T. Nawrot (Dept of Public Health, Occupational and Environmental Medicine, Lung Toxicology, Katholieke Universiteit Leuven, Leuven, Belgium) for his statistical advice.

\section{REFERENCES}

1 Yabuuchi E, Kosako Y, Oyaizu H, et al. Proposal of Burkholderia gen. nov. and transfer of seven species of the genus Pseudomonas homology group II to the new genus, with the type species Burkholderia cepacia (Palleroni and Holmes 1981) comb. nov. Microbiol Immunol 1992; 36: 1251-1275.

2 Sethi S, Murphy TF. Bacterial infection in chronic obstructive pulmonary disease in 2000: a state-of-the-art review. Clin Microbiol Rev 2001; 14: 336-363.

3 Qvarfordt I, Riise GC, Andersson BA, Larsson S. Lower airway bacterial colonization in asymptomatic smokers and smokers with chronic bronchitis and recurrent exacerbations. Respir Med 2000; 94: 881-887.

4 Cabello H, Torres A, Celis R, et al. Bacterial colonization of distal airways in healthy subjects and chronic lung disease: a bronchoscopic study. Eur Respir J 1997; 10: 1137-1144.

5 Lee TW, Brownlee KG, Conway SP, Denton M, Littlewood JM. Evaluation of a new definition for chronic Pseudomonas aeruginosa infection in cystic fibrosis patients. J Cyst Fibros 2003; 2: 29-34.

6 Proesmans M, Balinska-Miskiewicz W, Dupont L, et al. Evaluating the "Leeds criteria" for Pseudomonas aeruginosa infection in a cystic fibrosis centre. Eur Respir J 2006; 27: 937-943.

7 Nunley DR, Grgurich W, Iacono AT, et al. Allograft colonization and infections with pseudomonas in cystic fibrosis lung transplant recipients. Chest 1998; 113: 1235-1243.

8 de Pablo A, López S, Ussetti P, et al. [Lung transplant therapy for suppurative diseases.] Arch Bronconeumol 2005; 41: 255-259.

9 Bonvillain RW, Valentine VG, Lombard G, LaPlace S, Dhillon G, Wang G. Post-operative infections in cystic fibrosis and non-cystic fibrosis patients after lung transplantation. J Heart Lung Transplant 2007; 26: 890-897.

10 Schaedel C, de Monestrol, I, Hjelte L, et al. Predictors of deterioration of lung function in cystic fibrosis. Pediatr Pulmonol 2002; 33: 483-491.

11 Lechtzin N, John M, Irizarry R, Merlo C, Diette GB, Boyle MP. Outcomes of adults with cystic fibrosis infected with antibiotic-resistant Pseudomonas aeruginosa. Respiration 2006; 73: 27-33.

12 Jones AM, Dodd ME, Govan JR, et al. Burkholderia cenocepacia and Burkholderia multivorans: influence on survival in cystic fibrosis. Thorax 2004; 59: 948-951. 
13 Greene CM, Carroll TP, Smith SG, et al. TLR-induced inflammation in cystic fibrosis and non-cystic fibrosis airway epithelial cells. J Immunol 2005; 174: 1638-1646.

14 Tseng J, Do J, Widdicombe JH, Machen TE. Innate immune responses of human tracheal epithelium to Pseudomonas aeruginosa flagellin, TNF- $\alpha$, and IL-1 $\beta$. Am J Physiol Cell Physiol 2006; 290: C678-C690.

15 Delgado MA, Poschet JF, Deretic V. Nonclassical pathway of Pseudomonas aeruginosa DNA-induced interleukin-8 secretion in cystic fibrosis airway epithelial cells. Infect Immun 2006; 74: 2975-2984.

16 Tosi MF, van Heeckeren A, Ferkol TW, Askew D, Harding CV, Kaplan JM. Effect of Pseudomonas-induced chronic lung inflammation on specific cytotoxic T-cell responses to adenoviral vectors in mice. Gene Ther 2004; 11 : 1427-1433.

17 Vos R, Vanaudenaerde BM, Dupont LJ, Van Raemdonck DE, Verleden GM. Transient airway colonization is associated with airway inflammation after lung transplantation. Am J Transplant 2007; 7: 1278-1287.

18 Firoved AM, Ornatowski W, Deretic V. Microarray analysis reveals induction of lipoprotein genes in mucoid Pseudomonas aeruginosa: implications for inflammation in cystic fibrosis. Infect Immun 2004; 72: 5012-5018.

19 Trulock EP, Edwards LB, Taylor DO, et al. Registry of the International Society for Heart and Lung Transplantation: twenty-third official adult lung and heart-lung transplantation report-2006. J Heart Lung Transplant 2006; 25: 880-892.

20 De Soyza A, Archer L, Wardle J, et al. Pulmonary transplantation for cystic fibrosis: pre-transplant recipient characteristics in patients dying of peri-operative sepsis. J Heart Lung Transplant 2003; 22: 764-769.

21 Dobbin C, Maley M, Harkness J, et al. The impact of panresistant bacterial pathogens on survival after lung transplantation in cystic fibrosis: results from a single large referral centre. J Hosp Infect 2004; 56: 277-282.

22 Botha P, Archer L, Anderson RL, et al. Colonization of lung transplant recipients with Pseudomonas aeruginosa and the development of bronchiolitis obliterans syndrome. J Heart Lung Transplant 2006; 25: Suppl. 1, 82.

23 Yousem SA, Berry GJ, Cagle PT, et al. Revision of the 1990 working formulation for the classification of pulmonary allograft rejection: Lung Rejection Study Group. J Heart Lung Transplant 1996; 15: 1-15.

24 Standardization of Spirometry - 1987 Update. Statement of the American Thoracic Society. Am Rev Respir Dis 1987; 136: 1285-1298.

25 Estenne M, Maurer JR, Boehler A, et al. Bronchiolitis obliterans syndrome 2001: an update of the diagnostic criteria. J Heart Lung Transplant 2002; 21: 297-310.

26 Hachem RR, Chakinala MM, Yusen RD, et al. The predictive value of bronchiolitis obliterans syndrome stage 0-p. Am J Respir Crit Care Med 2004; 169: 468-472.

27 Sharples LD, McNeil K, Stewart S, Wallwork J. Risk factors for bronchiolitis obliterans: a systematic review of recent publications. J Heart Lung Transplant 2002; 21: 271-281.

28 Cook DJ, Fitzgerald JM, Guyatt GH, Walter S. Evaluation of the protected brush catheter and bronchoalveolar lavage in the diagnosis of nosocomial pneumonia. J Intensive Care Med 1991; 6: 196-205.
29 Mueller EW, Wood GC, Kelley MS, Boucher BA, Fabian TC, Croce MA. The predictive value of preliminary bacterial colony counts from bronchoalveolar lavage in critically ill trauma patients. Am Surg 2003; 69: 749-755.

30 Chan CC, Abi-Saleh WJ, Arroliga AC, et al. Diagnostic yield and therapeutic impact of flexible bronchoscopy in lung transplant recipients. J Heart Lung Transplant 1996; 15: 196-205.

31 Rust M, Albera C, Carratu L, et al. The clinical use of BAL in patients with pulmonary infections. Eur Respir J 1990; 3: 954-959.

32 Belda J, Cavalcanti M, Ferrer M, et al. Bronchial colonization and postoperative respiratory infections in patients undergoing lung cancer surgery. Chest 2005; 128: 1571-1579.

33 Soler N, Ewig S, Torres A, Filella X, Gonzalez J, Zaubet A. Airway inflammation and bronchial microbial patterns in patients with stable chronic obstructive pulmonary disease. Eur Respir J 1999; 14: 1015-1022.

34 Van Delden C, Iglewski BH. Cell-to-cell signaling and Pseudomonas aeruginosa infections. Emerg Infect Dis 1998; 4: 551-560.

35 Wilson R, Dowling RB. Lung infections. 3. Pseudomonas aeruginosa and other related species. Thorax 1998; 53: 213-219.

36 Chambers CE, Visser MB, Schwab U, Sokol PA. Identification of $\mathrm{N}$-acylhomoserine lactones in mucopurulent respiratory secretions from cystic fibrosis patients. FEMS Microbiol Lett 2005; 244: 297-304.

37 Wolfgang MC, Jyot J, Goodman AL, Ramphal R, Lory S. Pseudomonas aeruginosa regulates flagellin expression as part of a global response to airway fluid from cystic fibrosis patients. Proc Natl Acad Sci USA 2004; 101: 6664-6668.

38 Erickson DL, Endersby R, Kirkham A, et al. Pseudomonas aeruginosa quorum-sensing systems may control virulence factor expression in the lungs of patients with cystic fibrosis. Infect Immun 2002; 70: 1783-1790.

39 Middleton B, Rodgers HC, Cámara M, Knox AJ, Williams P, Hardman A. Direct detection of $N$-acylhomoserine lactones in cystic fibrosis sputum. FEMS Microbiol Lett 2002; 207: 1-7.

40 Ward C, Camara M, Forrest I, et al. Preliminary findings of quorum signal molecules in clinically stable lung allograft recipients. Thorax 2003; 58: 444-446.

41 Gillis RJ, White KG, Choi KH, Wagner VE, Schweizer HP, Iglewski BH. Molecular basis of azithromycin-resistant Pseudomonas aeruginosa biofilms. Antimicrob Agents Chemother 2005; 49: 3858-3867.

42 Hooi DS, Bycroft BW, Chhabra SR, Williams P, Pritchard DI. Differential immune modulatory activity of Pseudomonas aeruginosa quorum-sensing signal molecules. Infect Immun 2004; 72: 6463-6470.

43 Zimmermann S, Wagner C, Müller W, et al. Induction of neutrophil chemotaxis by the quorum-sensing molecule $N$ (3-oxododecanoyl)-L-homoserine lactone. Infect Immun 2006; 74: 5687-5692.

44 Lewenza S, Visser MB, Sokol PA. Interspecies communication between Burkholderia cepacia and Pseudomonas aeruginosa. Can J Microbiol 2002; 48: 707-716.

45 Angrill J, Agusti C, De Celis R, et al. Bronchial inflammation and colonization in patients with clinically stable bronchiectasis. Am J Respir Crit Care Med 2001; 164: 1628-1632. 
46 Vanaudenaerde BM, Wuyts WA, Dupont LJ, Van Raemdonck DE, Demedts MM, Verleden GM. Interleukin17 stimulates release of interleukin- 8 by human airway smooth muscle cells in vitro: a potential role for interleukin-17 and airway smooth muscle cells in bronchiolitis obliterans syndrome. J Heart Lung Transplant 2003; 22: 1280-1283.

47 Verleden GM, Vanaudenaerde BM, Dupont LJ, Van Raemdonck DE. Azithromycin reduces airway neutrophilia and interleukin-8 in patients with bronchiolitis obliterans syndrome. Am J Respir Crit Care Med 2006; 174: 566-570.

48 Reynaud-Gaubert M, Marin V, Thirion X, et al. Upregulation of chemokines in bronchoalveolar lavage fluid as a predictive marker of post-transplant airway obliteration. $J$ Heart Lung Transplant 2002; 21: 721-730.

49 Holzmann D, Speich R, Kaufmann T, et al. Effects of sinus surgery in patients with cystic fibrosis after lung transplantation: a 10-year experience. Transplantation 2004; 77: 134-136.

50 Vanaudenaerde BM, Wuyts WA, Geudens $\mathrm{N}$, et al. Macrolides inhibit IL17-induced IL8 and 8-isoprostane release from human airway smooth muscle cells. Am J Transplant 2007; 7: 76-82.

51 Rasmussen TB, Givskov M. Quorum sensing inhibitors: a bargain of effects. Microbiology 2006; 152: 895-904.

52 Tateda K, Comte R, Pechere JC, Köhler T, Yamaguchi K, Van Delden C. Azithromycin inhibits quorum sensing in Pseudomonas aeruginosa. Antimicrob Agents Chemother 2001; 45: 1930-1933.

53 Nalca Y, Jänsch L, Bredenbruch F, Geffers R, Buer J, Häussler S. Quorum-sensing antagonistic activities of azithromycin in Pseudomonas aeruginosa PAO1: a global approach. Antimicrob Agents Chemother 2006; 50: 1680-1688.

54 Hurst JR, Wilkinson TM, Perera WR, Donaldson GC, Wedzicha JA. Relationships among bacteria, upper airway, lower airway, and systemic inflammation in COPD. Chest 2005; 127: 1219-1226.

55 Hadjiliadis D, Chaparro C, Gutierrez C, et al. Impact of lung transplant operation on bronchiolitis obliterans syndrome in patients with chronic obstructive pulmonary disease. Am J Transplant 2006; 6: 183-189. 